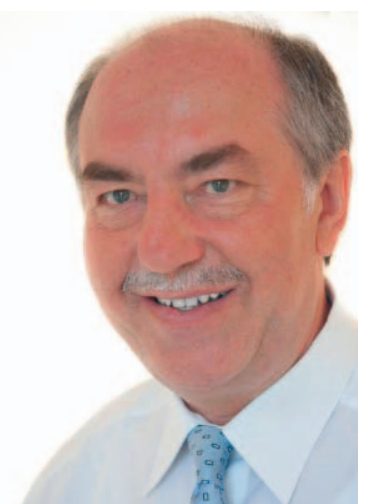

Prof. Dr. med. Harald Meden, Richterswil

\section{Ganzheitliche Gynäkologie}

\section{Konzept der WHO}

Im Jahr 2002 stellte der damalige Direktor der Weltgesundheitsorganisation (WHO), Jonathan Glick, eine neue Initiative der WHO vor: Mehr Anerkennung für Naturheilverfahren und Komplementärmedizin. Ziel dieser Initiative war es, Schulmedizin durch Naturheilverfahren und Komplementärmedizin zu ergänzen. Als Plan für die Umsetzung wurde die Sammlung von Nachweisen zur Wirksamkeit, Qualität und Sicherheit von Massnahmen der Naturheilkunde und Komplementärmedizin propagiert. Als weiterer Weg zum Ziel sollten bewährte Alternativen $\mathrm{zu}$ schulmedizinischen Behandlungen von den staatlichen Gesundheitssystemen anerkannt und gefördert werden.

Weltweit sind in den zurückliegenden 10 Jahren zahlreiche Fortschritte in dem oben genannten Sinn zu verzeichnen. Die SchWEIZERISCHE ZEITSChrIfT FÜR GANZHEITSMEDIZIN leistet auf ihre Weise seit vielen Jahren einen wichtigen Beitrag zur Umsetzung dieser Initiative der WHO.

\section{Ganzheitsmedizin in der Gynäkologie und Geburtshilfe}

Der Einsatz von Naturheilverfahren und Komplementärmedizin bei Beschwerden während der Schwangerschaft, unter der Geburt und in der Stillzeit hat eine lange Tradition, sowohl in der Selbstbehandlung als auch in der Behandlung durch Fachpersonen. Dies gilt insbesondere für die Tätigkeit von Frauenärztinnen und Frauenärzten sowie für Hebammen. Mit Blick auf das Ungeborene und auf das Neugeborene sollen Behandlungsrisiken minimiert werden; somit können viele klassische schulmedizinische Behandlungsformen in diesen besonderen Lebensphasen nicht eingesetzt werden. Dieser Grundsatz setzt sich dann zum Teil in der Behandlung in der Kinderheilkunde fort.

Als aktuelles Beispiel zum Einsatz von Naturheilverfahren beeindrucken die überzeugenden wissenschaftlichen Daten zur Wirksamkeit von Bryophyllum bei der Behandlung von Schlafstörungen bei Schwangeren. Diese neuen Erkenntnisse wurden mithilfe von schulmedizinischen Methoden erarbeitet und unter anderem im Juni 2011 auf dem Kongress der Schweizer Gesellschaft für Gynäkologie und Geburtshilfe vorgestellt.

Auch die Gynäkologie ist eine Domäne für naturheilkundliche und komplementäre Therapien. Die Behandlung von Beschwerden in den Wechseljahren ist ein gutes Beispiel hierfür. Gerade diese Indikation hat in den letzten Jahren deutlich an Bedeutung gewonnen, nachdem die Verschreibung von Hormonersatztherapien nach der Brustkrebsdiskussion deutlich zurückgegangen ist.

Die Behandlung von Menstruationsbeschwerden, Harnwegsinfekten, Scheidenentzündungen und Endometriosebeschwerden sind weitere Beispiele für den wachsenden Einsatz von Naturheilverfahren und Komplementärmedizin.

\section{Ganzheitliche Gynäkologische Onkologie}

Der Tenor auf dem letzten Kongress der Amerikanischen Gesellschaft für klinische Onkologie (ASCO) lautete: individuelle und risikoadaptierte onkologische Therapie. Dies bezog sich in erster Linie auf klinische Risiken wie Tumorgrösse, Metastasen und Lymphknotenbefall sowie auf histopathologische und molekularbiologische Kriterien.

Die meisten Patientinnen mit Brustkrebs setzen - ergänzend zur schulmedizinischen Behandlung - zusätzlich komplementärmedizinische Massnahmen ein, sei es mit professioneller Unterstützung oder ohne, und haben auf diese Weise ihr individuelles ganzheitliches medizinisches Konzept auf einer der Molekularbiologie übergeordneten Ebene. Für Patientinnen mit anderen gynäkologischonkologischen Erkrankungen gilt dies in ähnlicher Weise.

So gestalten sich viele Patientinnen ihr individuelles Konzept, bestehend aus Schulmedizin plus Elementen der

\section{KARGER}

Fax +497614520714 Information@Karger.d www.karger.com
Prof. Dr. med. Harald Meden

Spezialarzt Gynäkologie und Geburtshilfe FMH

Leitender Arzt Operative Medizin, Paracelsus-Spital Richterswil

Bergstrasse 16, 8805 Richterswil, Schweiz

Tel. +41 44 787-2920, Fax -2940

harald.meden@paracelsus-spital.ch 
Komplementärmedizin, und haben damit ihre persönliche Ganzheitsmedizin. Eine wichtige Indikation ist in diesem Zusammenhang die Reduktion von Nebenwirkungen schulmedizinischer Behandlungen, insbesondere postoperativ, während der Chemotherapie und während einer Strahlentherapieserie. Dies ist auch ein wichtiger Beitrag, um dem Abbruch schulmedizinischer Therapien entgegenzuwirken.

Für viele Menschen mit einer Krebserkrankung ist es wichtiger, den nach der Diagnosestellung verbleibenden Jahren mehr Leben zu geben als dem verbleibenden Leben mehr Jahre. Damit steht die Lebensqualität zunehmend im Zentrum des Interesses von Patienten und Therapeuten. Auch in diesem Bereich haben Komplementärmedizin und Naturheilverfahren eine wachsende Bedeutung.

\section{Annäherung in der Wissenschaft}

Schulmedizin auf der einen Seite und Naturheilverfahren und Komplementärmedizin auf der anderen Seite waren lange Zeit gegensätzliche Pole, sodass man sich bekämpfte. Dann gab es eine Phase des Stillhaltens und der Duldung, zumindest in begrenztem Masse. In der aktuellen Entwicklung gibt es den Beginn eines Dialogs über Gemeinsamkeiten und Unterschiede, Messtechniken und Methoden. Mithilfe dieser Elemente ist im Moment eine wissenschaftliche Annäherung zu beobachten. Ein Beispiel hierfür: der Fragebogen QLQ-C30 der EORTC (European Organisation for Research and Treatment of Cancer). Er dient seit 2 Jahrzehnten der Erfassung der Lebensqualität von onkologischen Patientinnen in schulmedizinischen onkologischen Therapiestudien, nachdem erkannt wurde, dass zur Beurteilung neuer Medikamente und Konzepte in der Onkologie nicht allein Überlebenszeiten, Rezidivraten und Laborparameter wichtig sind, sondern in besonderem Masse die Erfassung der Lebensqualität. Dieser Fragebogen wurde nun in der jüngsten Vergangenheit auch in komplementärmedzinischen Studien zur Erfassung der Lebensqualität von Mammakarzinom-Patientinnen während einer Chemotherapie mit und ohne begleitender Misteltherapie eingesetzt, was zu einer deutlichen Aufwertung der Studienqualität und zur besseren Akzeptanz der Ergebnisse geführt hat. Details hierzu sind in der Publikation von Simões-Wüst et al. in dieser Ausgabe der SchweizeRISCHEN ZEITSCHRIFT FÜR GANZHEITSMEDIZIN zu lesen.

Ein weiteres Beispiel für die Annäherung in der Wissenschaft ist die zunehmende Etablierung von Lehraufträgen und Lehrstühlen für Naturheilverfahren, Komplementärmedizin sowie Traditionelle Medizin an den Universitäten. Auch Fortbildungskurse für klinisch und praktisch tätige Ärzte werden zunehmend als Zertifikatkurse wissenschaftlicher Fachgesellschaften und an Universitäten angeboten, unter anderem mit dem Ziel, die ärztliche Beratungskompetenz auf dem Gebiet der Ganzheitlichen Gynäkologischen Onkologie in den Sprechstunden zu erhöhen (www.natum.de/ggo).

Die genannten Punkte zeigen, dass die am Anfang beschriebe Initiative der WHO zunehmend umgesetzt wird. In diesem Sinne wünsche ich Ihnen eine spannende Lektüre der vor Ihnen liegenden Ausgabe mit dem Fokus «Gynäkologie». 